\title{
Are Dawn Collections of Anopheles stephensi a Better Method To Estimate the Resting Vector Density? A Study from Chennai, India
}

\author{
Sangamithra Ravishankaran, ${ }^{1,2}$ Aswin Asokan, ${ }^{1}$ N. A. Johnson Amala Justin, ${ }^{3}$ Shalu Thomas, ${ }^{1}$ Manu Thomas Mathai, ${ }^{2}$ and \\ Alex Eapen ${ }^{1^{*}}$ \\ ${ }^{1}$ ICMR - National Institute of Malaria Research, Field Unit, Chennai, India; ${ }^{2}$ Department of Zoology, Madras Christian College, Chennai, India; \\ ${ }^{3}$ Regional Office for Health and Family Welfare, Chennai, India
}

\begin{abstract}
The study was an attempt to capture Anopheles stephensi from cattle sheds during dawn to understand the realistic density of the resting mosquitoes. A2-year longitudinal study was carried out in cattle sheds in close proximity to the human dwellings to collect the resting vector mosquitoes. The man-hour density of An. stephensi ranged from 24.7 to 206.5 . The vector incrimination results indicated $0.15 \%$ of $A n$. stephensi infected with Pv210 in 2015 and $0.09 \%$ in 2016 . The current study indicated that cattle sheds are still the preferred resting place of $A n$. stephensi and that dawn is the perfect time to collect and estimate its densities. Hence, adult vector control may also be given due importance in addition to the routine larval source management measures to curb malaria transmission in an urban setting.
\end{abstract}

Malaria is an infectious and sometimes fatal disease occurring in many tropical and subtropical regions. Malaria is caused by Plasmodium, a protozoan parasite, and is transmitted by female Anopheles mosquitoes. Plasmodium vivax and Plasmodium falciparum are the predominant malaria parasite species observed in the Indian subcontinent. In an urban setting, the collection of adult vectors is an uphill task owing to various reasons, such as the compounded spatial heterogeneity, complexity in feeding, and resting preferences, unlike the immature forms. ${ }^{1}$ Hence, it is relevant to have actual estimates of adult vector density to plan appropriate vector control interventions. The study was conducted in Besant Nagar $\left(13.0002^{\circ} \mathrm{N}, 80.2668^{\circ} \mathrm{E}\right)$, a residential area with slums adjoining the seashore in the southeastern part of Chennai, characterized by the perennial transmission of malaria, predominantly $P$. vivax. ${ }^{1-3}$ The vector responsible for the transmission of urban malaria in Chennai is Anopheles stephensi, which breed mainly in clear and stored water habitats. ${ }^{1}$ Adult vector control measures, such as indoor residual spraying, are neither in practice nor in the control program in urban cities and towns. The habitats missed by the larvicide application during the routine weekly surveillance will support vector breeding followed by emergence, and the vectors will find cryptic places to hide. This differs from houses, where people often use personal protection measures that force the vectors to seek shelter and blood meals elsewhere and bite animals tied in cattle sheds and human beings who are exposed outdoors without proper attire. Earlier collections undertaken at dusk could only get a few vector mosquitoes ${ }^{2}$ and do not represent the actual resting phase because the vectors were still seeking the host. Hence, an attempt was made to screen and capture the vector mosquitoes resting in cattle sheds at dawn. Thus, the study site was selected based on the malaria prevalence of the area and vector breeding potential in view of the various longitudinal studies undertaken simultaneously in the same site. ${ }^{1-6}$

*Address correspondence to Alex Eapen, ICMR - National Institute of Malaria Research, NIE Campus, 2nd Main Road, TNHB, Ayapakkam, Chennai- 600 077, India. E-mail: alexeapen@yahoo. com
The resting dawn collections were undertaken in cattle sheds from January 2015 to December 2016 to capture most of the resting An. stephensi. Cattle sheds were surveyed every week during dawn (4:30-06:00 AM) using a flashlight and an oral/mouth aspirator. The sheds were selected depending on the accessibility and on the experience of a previous longitudinal survey. ${ }^{2}$ A total of 17 cattle sheds were surveyed; six cattle sheds were surveyed on a regular basis (sentinel), whereas others were surveyed randomly. In each cattle shed, 15-30 minutes were spent depending on the size and area and the presence or absence of mosquitoes at the time of collection. The collected mosquitoes were brought alive to the laboratory and identified to the species level by referring to the standard mosquito identification keys. ${ }^{7,8}$ The female mosquitoes were enumerated based on their abdominal conditions. The late-stage fed, semi-gravid, and gravid appearance of the abdomen was considered as resting stages, and the unfed guts and/or freshly fed were considered as feeding stages. ${ }^{9,10}$ The man-hour density (MHD) of An. stephensi was estimated by dividing the total number of female mosquitoes collected by total time spent within a 1-hour period. ${ }^{2}$ The mosquitoes were then processed using circumsporozoite sandwich ELISA following the Malaria Research and Reference Reagent Resource Center (MR4) protocol. ${ }^{11}$ The monthly MHD of An. stephensi in cattle sheds was plotted against corresponding malaria incidence data obtained from the Regional Office for Health and Family Welfare, Chennai, and rainfall data were obtained from India Meteorological Department, Chennai (Figure 1).

Resting dawn collections undertaken are shown in Table 1. A total of 214 dawn collections were made in 2015 and 206 in 2016, which resulted in the capture of 2,386 (MHD: 45.7) and 5,759 (MHD: 115.7) female An. stephensi, respectively. Vector incrimination of female $A n$. stephensi revealed $0.15 \%$ and $0.09 \%$ of infection with Pv210 in 2015 and 2016, respectively (Table 1). The reduction in vector incrimination despite the high vector density was reflected in the declining malaria prevalence in the study site compared with the previous years (Figure 1). This may also be due to the low parasite load in the community, which denies the chance of getting parasitized blood from relatively few infected individuals. The adult vector density ranged from 24.7 to 206.5 and peaked during July 2016. Man-hour density was high in 2016 owing to heavy 


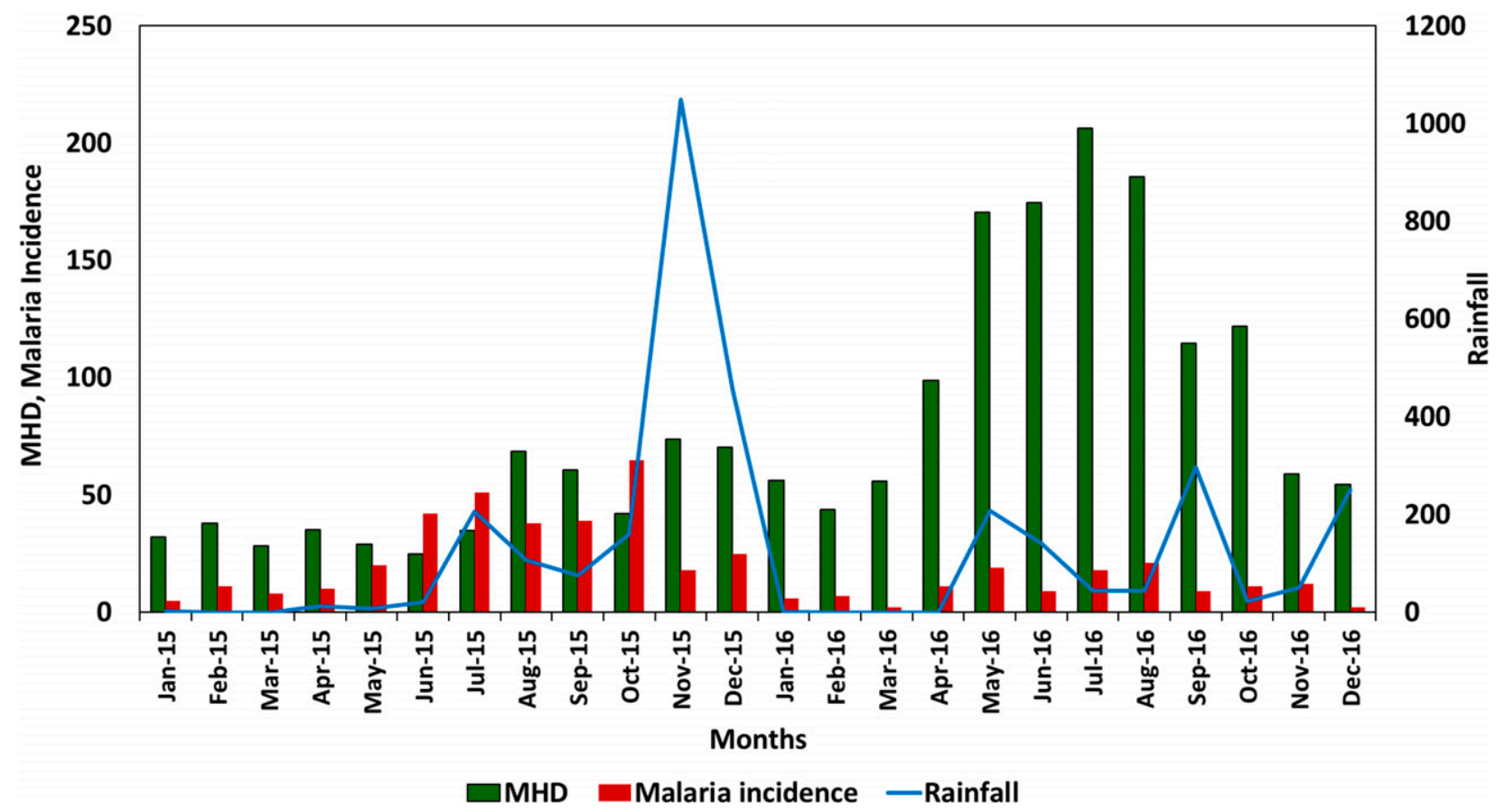

FIGURE 1. Monthly man-hour density in cattle sheds with corresponding malaria incidence and rainfall. This figure appears in color at www. ajtmh.org.

rainfall (cyclone) during the end of 2015 and again during the middle of 2016 (Figure 1). The composition of other mosquito species captured besides An. stephensi in cattle sheds is illustrated in Figure 2. Culex gelidus was the predominant species (44.63\%), followed by An. stephensi (38.14\%) and Culex quinquefasciatus (16.23\%). Other mosquito species, such as Armigeres subalbatus, Aedes aegypti, Anopheles subpictus, Anopheles vagus, Anopheles barbirostris, Anopheles annularis, and Mansonia annulifera, were collected in negligible numbers (1\%).

The present study analyzed the density of adult vectors resting during dawn in an urban slum setting. During monsoon season, An. stephensi also breeds in roof gutters and on roof tops (terrace) in stagnant rainwater collections, which leads to breeding and the resultant density appended to the vector density from the potential breeding habitats. In urban areas, although $A n$. stephensi is present throughout the year, the density peaks during the rainfall period. ${ }^{12}$ Chennai experienced devastating floods between November and December 2015, which could have contributed to the surge in vector breeding. ${ }^{13}$ The increased density of mosquitoes captured could be attributed to the collection time because there is less disturbance in the early morning period, and hence the mosquitoes could rest for a longer period. Consequently, adult vector collections during dawn would provide actual estimates of resting vector mosquitoes in cattle sheds. The test on the susceptibility status of the adult An. stephensi collected from the cattle sheds during the study period with the recommended dosage of deltamethrin $(10 \mu \mathrm{g})$ following CDC Bottle assay guidelines ${ }^{14}$ indicated $100 \%$ mortality. Factors such as the vast number of overhead tanks with unapproachable and dilapidated water storage tanks, thatched roof structures, and cattle sheds in close proximity to the houses are the characteristic of the study site. ${ }^{1-3,15}$ Therefore, vector intervention measures need to be situation specific depending on the area, and the presence of these factors must be periodically reviewed for successful and effective implementation.

One of the limitations of the study was that parallel dusk collection data could not be generated due to the lack of manpower for undertaking collections simultaneously and community refusal by disturbing the limited privacy especially in a busy metropolitan city like Chennai. Blood meal preference of the vector mosquitoes was not checked in the present

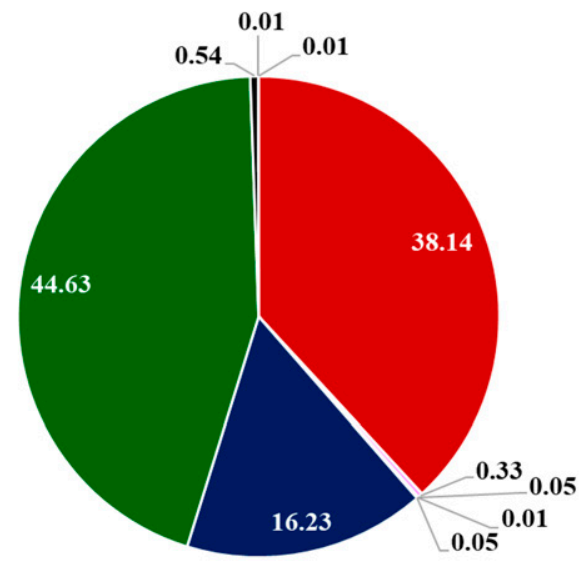

\footnotetext{
- Anopheles stephensi (38.14\%)

" Anopheles subpictus (0.33\%)

- Anopheles vagus (0.05\%)

- Anopheles barbirostris (0.01\%)

- Anopheles annularis (0.05\%)

- Culex gelidus (44.63\%)

- Culex quinquefasciatus (16.23\%)

- Armigeres subalbatus (0.54\%)

- Aedes aegypti (0.01\%)

- Mansonia annulifera (0.01\%)
}

FlgURE 2. Mosquito species composition in cattle sheds. This figure appears in color at www.ajtmh.org. 


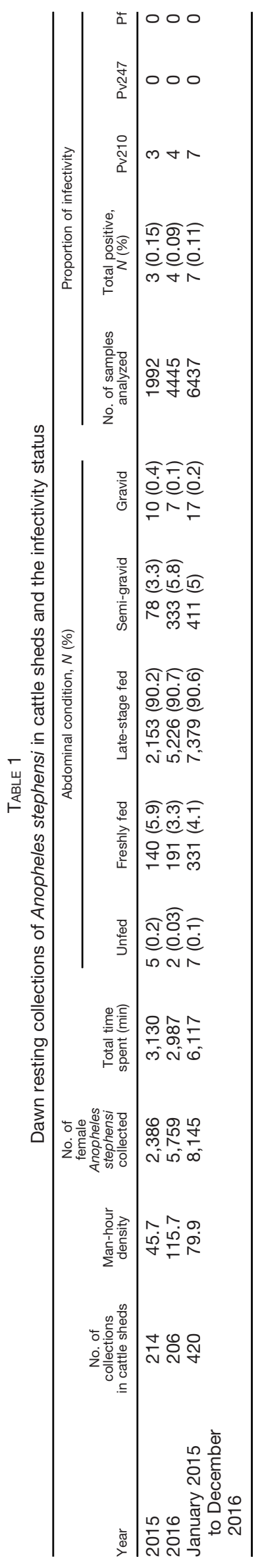

study. Nevertheless, a study undertaken during the previous year from the same site indicated $95 \%$ of the analyzed An. stephensi to have engorged with bovine blood, $0.9 \%$ with human blood; the remaining were unknown (neither bovine nor human). ${ }^{2}$ Thus, the study elucidates the findings to plan vector intervention policies directed toward sustainable vector control strategies.

The present study has disclosed that cattle sheds are still the preferred resting place ${ }^{2}$ of the vector mosquitoes over human dwellings with intense repellent pressure. The dawn collections revealed the actual estimates of resting vector mosquitoes, also where and when to plan adult vector surveillance. Intervention measures such as targeted spray in cattle sheds in close proximity to human dwellings may be a control measure in malaria endemic areas with profound vector breeding, but this should be done with extreme caution. Precautionary measures need to be taken to ensure a targeted spray that will not drive the vectors to human dwellings as a result of behavioral changes due to insecticides. In general, houses may be fixed with window and door insect screens to prevent entry of vector mosquitoes. LLIN distribution is also an important vector control measure to households that are at risk or in areas where vector is abundant as well as in malaria endemic areas. Hence, in addition to the existing larval source management in urban areas, adult vector control may be intensified to prevent vector bites and to curb malaria transmission.

Received July 9, 2020. Accepted for publication April 28, 2021.

Published online June 28, 2021.

Acknowledgments: We thank the National Institute of Malaria Research and Indian Council of Medical Research for providing the necessary facilities and support; Drs. Amit Sharma (Director, ICMRNational Institute of Malaria Research), Neena Valecha (Former Director, ICMR-National Institute of Malaria Research), Jane Carlton (New York University), and Matthew B. Thomas (Penn State University) for their valuable suggestions and support; the staff of the NIMR field unit, Chennai, and technical staff of Regional office for Health and Family Welfare (Govt. of India) at Besant Nagar, Chennai; the communities of Adyar, Besant Nagar, and Thiruvanmiyur for permitting us to carry out the survey in their premises; and the reviewers for their critical review that changed the focus and quality of the manuscript. The American Society of Tropical Medicine and Hygiene (ASTMH) assisted with publication expenses.

Financial support: The work was supported by the National Institute of Allergy and Infectious Diseases, NIH Grant U19AI089676. The content of this manuscript is solely the responsibility of the authors and does not necessarily represent the official views of the NIH.

Authors' addresses: Sangamithra Ravishankaran, Aswin Asokan, and Shalu Thomas, ICMR - National Institute of Malaria Research, Field Unit, NIE Campus, Ayapakkam, Chennai, India, E-mails: vr. sangamithra@gmail.com, ashwinviro@gmail.com, and jyshalu@ gmail.com. N. Johnson Amala Justin, Regional Office for Health and Family Welfare, Rajaji Bhavan, Besant Nagar, Chennai, India, E-mail: johnsonamalajustin@gmail.com. Manu Thomas Mathai, Department of Zoology, Madras Christian College, Tambaram, Chennai, India, E-mail: manuthomasmcc@gmail.com.

\section{REFERENCES}

1. Thomas S, Ravishankaran S, Justin NJ, Asokan A, Mathai MT, Valecha N, Thomas MB, Eapen A, 2016. Overhead tank is the potential breeding habitat of Anopheles stephensi in an urban transmission setting of Chennai, India. Malar J 15: 274.

2. Thomas S, Ravishankaran S, Justin NJ, Asokan A, Mathai MT, Valecha N, Montgomery J, Thomas MB, Eapen A, 2017. 
Resting and feeding preferences of Anopheles stephensi in an urban setting, perennial for malaria. Malar J 16: 111.

3. Thomas S, Ravishankaran S, Justin NJ, Asokan A, Kalsingh TM, Mathai MT, Valecha N, Montgomery J, Thomas MB, Eapen A, 2018. Microclimate variables of the ambient environment deliver the actual estimates of the extrinsic incubation period of Plasmodium vivax and Plasmodium falciparum: a study from a malaria-endemic urban setting, Chennai in India. Malar J 17: 201.

4. Van Eijk AM et al., 2016. What is the value of reactive case detection in malaria control? A case-study in India and a systematic review. Malar J 15: 67.

5. Van Eijk AM et al., 2016. The use of mosquito repellents at three sites in India with declining malaria transmission: surveys in the community and clinic. Parasit Vectors 9: 418.

6. Van Eijk AM et al., 2019. The burden of submicroscopic and asymptomatic malaria in India revealed from epidemiology studies at three varied transmission sites in India. Sci Rep 9: 1-1.

7. Nagpal BN, Srivastava A, Saxena R, Ansari MA, Dash AP, Das SC, 2005. Pictorial Identification Key for Indian Anophelines. Delhi, India: Malaria Research Centre.

8. Nagpal BN, Sharma VP, 1995. Indian Anophelines. New Delhi, India: Oxford and IBH Publishing Co. Pvt Ltd.
9. WHO, 1975. Manual on Practical Entomology in Malaria: Methods and Techniques, Part 2. Geneva, Switzerland: WHO Division of Malaria Other Parasitic Diseases.

10. Basseri HR, Raeisi A, Khakha MR, Pakarai A, Abdolghafar $H$, 2010. Seasonal abundance and host-feeding patterns of anopheline vectors in malaria endemic area of Iran. $J$ Parasitol Res 2010: 671291.

11. Wirtz R, Avery M, Benedict M, 2007. Plasmodium sporozoite ELISA. MR4: 11

12. Dev V, Sharma VP, 2013. The dominant mosquito vectors of human malaria in India. Manguin S, ed. Anopheles mosquitoes: New insights into malaria vectors. IntechOpen.

13. Angeline N, Anbazhagan S, Surekha A, Joseph S, Kiran PR, 2017. Health impact of chennai floods 2015: observations in a medical relief camp. Int J Health Syst Disaster Manage 5: 46.

14. Brogdon W, Chan A, 2010. Guideline for Evaluating Insecticide Resistance in Vectors Using the CDC Bottle Bioassay. Atlanta, GA: CDC.

15. Thomas S, Ravishankaran S, Asokan A, Justin NJ, Kalsingh TM, Mathai MT, Valecha N, Eapen A, 2018. Socio-demographic and household attributes may not necessarily influence malaria: evidence from a cross sectional study of households in an urban slum setting of Chennai, India. Malar J 17: 4. 